\title{
Curvature and isocurvature perturbations from two-field inflation in a slow-roll expansion
}

\author{
Christian T. Byrnes and David Wands \\ Institute of Cosmology and Gravitation, University of Portsmouth, \\ Mercantile House, Portsmouth PO1 2EG, United Kingdom
}

(Dated: February 5, 2008)

\begin{abstract}
We calculate the power spectra of primordial curvature and isocurvature perturbations from a general two field inflation model at next-to-leading order correction in a slow-roll expansion. In particular we calculate the spectral indices to second order in slow-roll parameters. We show that the cross-correlation of the curvature and isocurvature perturbations at the time of Hubble-exit during inflation is non-zero at first-order in slow-roll parameters. We apply our results to different classes of inflation, including inflaton and curvaton scenarios. The spectrum of primordial gravitational waves, curvature and isocurvature perturbations obey generalised consistency relations in two-field inflation models. We give the first two consistency relations in an infinite hierarchy.
\end{abstract}

PACS numbers: $98.80 . \mathrm{Cq}$

astro-ph/0605679

\section{INTRODUCTION}

During inflation the vacuum fluctuations of light scalar fields grow into super-Hubble density perturbations which are believed to be the origin of the structure seen in the universe today. Single-field slow roll inflation predicts a nearly scale invariant spectrum of gaussian curvature perturbations. However from a particle physics point of view it is natural to expect there to be more then one scalar field rolling during inflation. During multiple field inflation a spectrum of isocurvature as well as curvature perturbations can be generated and the two may be correlated [1].

In this paper we consider the case of two field inflation with an arbitrary potential and arbitrary background trajectory. Following Ref. $[2,3]$ we introduce arbitrary transfer functions to parameterise the generation of primordial curvature and isocurvature perturbations from isocurvature perturbations during inflation. We present the power spectra of the isocurvature and curvature perturbations to first-order in slow roll as well as the cross-correlation, presenting the results in terms of the slow-roll parameters and the (in principle) observable correlation angle. We are then able to calculate the tilts of all three power spectra to second-order in slow-roll parameters (see also Ref. [4]). This generalises the result of Stewart and Lyth [5] for adiabatic perturbations from single-field inflation. For an alternative approach to calculating the primordial curvature perturbation in multi-field models (but not the isocurvature perturbation), based on the $\delta N$-formalism [6], see Ref. [7, 8].

In section II we calculate the scalar field perturbations during inflation, first performing a global rotation in field space to a basis where the perturbations are uncorrelated at Hubble exit. We then rotate to the local (instantaneous) basis of adiabatic and isocurvature perturbations $[4,9,10]$ and show that they are correlated at first-order in slow roll at Hubble exit. The only exception is the case of a straight background trajectory in field space, e.g. for a symmetric potential [11], when the local rotation is in fact a constant (global) rotation which coincides with the uncorrelated basis and hence the curvature and isocurvature perturbations are uncorrelated at Hubble exit.

In section III we change variables to the dimensionless curvature and isocurvature perturbations which are then related to the primordial curvature and isocurvature perturbations after inflation, and introduce the dimensionless correlation angle. The curvature and isocurvature perturbations are evolved on superhorizon scales by introducing two arbitrary transfer functions, which parameterise our ignorance of the detailed physics after Hubble exit and through the end of inflation and reheating. We present results for the power spectra and tilts including the next to leading order terms and also the running at leading order, which is second-order in slow roll. We also briefly present the power spectrum, tilt and running for tensor perturbations, which are unchanged from the single-field case [12]. In section IV we simplify the results to the interesting special cases of a straight background trajectory, which includes assisted inflation with exponential potentials [13-15], the curvaton scenario [16], and the inflaton scenario allowing for an additional uncorrelated isocurvature field present during inflation. We conclude in section V.

Appendices contain definitions of all the slow-roll parameters used and the relations between these parameters when defined in terms of the Hubble parameter or the potential, their derivatives and further details of the calculation of the tilts. Throughout this paper we use the notation that results accurate at first order in slow roll are denoted by $\simeq$, while equality at second order in slow roll is denoted by $\cong$. 


\section{INITIAL POWER SPECTRA}

\section{A. Background equations of motion}

We consider two scalar fields with Lagrange density

$$
\mathcal{L}=-\frac{1}{2} \sum_{I=1}^{2} g^{\mu \nu} \phi_{I, \mu} \phi_{I, \nu}-V\left(\phi_{1}, \phi_{2}\right)
$$

We thus allow for an arbitrary interaction potential, $V\left(\phi_{1}, \phi_{2}\right)$, but for simplicity consider only canonical kinetic terms. It should be possible to generalise our results to non-minimal kinetic terms, see for example [4, 10].

The background equations of motion are

$$
\begin{aligned}
& \ddot{\phi}_{I}+3 H \dot{\phi}_{I}+V_{I}=0 \\
& H^{2}=\frac{8 \pi G}{3}\left[V+\frac{1}{2}\left(\dot{\phi}_{1}^{2}+\dot{\phi}_{2}^{2}\right)\right],
\end{aligned}
$$

where $V_{I} \equiv \partial V / \partial \phi_{I}$. To solve these equations we use the slow roll approximation to rewrite them as

$$
\dot{\phi}_{I} \simeq-\frac{V_{I}}{3 H}\left(1+\frac{1}{3} \delta_{I}^{H}\right), \quad H^{2} \simeq \frac{8 \pi G}{3} V\left(1+\frac{1}{3} \epsilon^{H}\right)
$$

where $\delta_{I}^{H}$ and $\epsilon^{H}$ are slow-roll parameters as defined in appendix A, which we assume to be small. We make the standard slow roll assumption that the time derivative of the slow-roll parameters are higher order in slow roll.

\section{B. The perturbation equations}

The equations of motion for the perturbed fields in the spatially flat gauge are [17]

$$
\ddot{\delta} \phi_{I}+3 H \dot{\delta} \phi_{I}+\frac{k^{2}}{a^{2}} \delta \phi_{I}+\sum_{J}\left[V_{I J}-\frac{8 \pi G}{a^{3}} \frac{d}{d t}\left(\frac{a^{3}}{H} \dot{\phi}_{I} \dot{\phi}_{J}\right)\right] \delta \phi_{J}=0
$$

where $k$ is the comoving wavenumber. To simplify the above equations we change variables to $u_{I}=a \delta \phi_{I}$ and to conformal time $\tau$, defined by $d \tau=d t / a$. To lowest order in slow roll, $\tau \simeq-(1+\epsilon) /(a H)$, the equations of motion simplifies to

$$
u_{I}^{\prime \prime}+\left(k^{2}-\frac{2}{\tau^{2}}\right) u_{I}=\frac{3}{\tau^{2}} \sum_{J} M_{I J} u_{J}
$$

where the prime denotes a derivative with respect to conformal time and the interaction matrix is

$$
M_{I J} \simeq\left(\begin{array}{cc}
\epsilon^{H}+2 \epsilon_{11}^{H}-\eta_{11} & 2 \epsilon_{12}^{H}-\eta_{12} \\
2 \epsilon_{12}^{H}-\eta_{12} & \epsilon^{H}+2 \epsilon_{22}^{H}-\eta_{22}
\end{array}\right) .
$$

Note that the superscript $H$ denotes a slow-roll parameter defined in terms of the Hubble parameter, while all other slow-roll parameters are defined in terms of the potential (see appendix A).

The two differential equations for $u_{I}$ are coupled but they can be decoupled at Hubble-exit by performing a rotation to diagonalise the interaction matrix (7) to first order in slow roll. For previous discussions on how to solve the coupled equations (6) see, for example, $[10,18]$. The rotation matrix is given by

$$
U=\left(\begin{array}{cc}
\cos \Theta & -\sin \Theta \\
\sin \Theta & \cos \Theta
\end{array}\right)
$$

where the rotation angle $\Theta$ is defined by

$$
\tan 2 \Theta=2\left[\frac{2 \epsilon_{12}^{H}-\eta_{12}}{2\left(\epsilon_{11}^{H}-\epsilon_{22}^{H}\right)-\left(\eta_{11}-\eta_{22}\right)}\right]_{*} .
$$


The subscript $*$ refers to a quantity evaluated at horizon crossing, $k=a_{*} H_{*}$. For a given wavenumber $k$, $\Theta$ is a constant, but there is a $k$ dependence since the slow roll parameters are evaluated at horizon crossing and each wave mode has a different horizon crossing time. Note that since the rotation angle $\Theta$ is defined in terms of a ratio of slowroll parameters it is in general not small. If all of the slow-roll parameters become arbitrarily small Eq. (9) becomes ambiguous, because the equations of motion (6) are then decoupled to first order in any basis and any rotation will work. The rotation matrix (8) satisfies

$$
U^{\dagger} M U=\operatorname{diag}\left(\lambda_{1}, \lambda_{2}\right)
$$

where

$$
\lambda_{1,2} \simeq \frac{1}{2}\left[4 \epsilon^{H}-\left(\eta_{11}+\eta_{22}\right) \pm \sqrt{\left[2\left(\epsilon_{11}^{H}-\epsilon_{22}^{H}\right)-\left(\eta_{11}-\eta_{22}\right)\right]^{2}+4\left(2 \epsilon_{12}^{H}-\eta_{12}\right)^{2}}\right]_{*} .
$$

Denoting the decoupled variables by $v_{I}$, we have

$$
u_{I}=\sum_{J} U_{I J} v_{J}
$$

Therefore left-multiplying (6) by $U^{\dagger}$ and rewriting in the $v_{I}$ basis we have

$$
v_{I}^{\prime \prime}+\left(k^{2}-\frac{1}{\tau^{2}}\left(\mu_{I}^{2}-\frac{1}{4}\right)\right) v_{I} \simeq 0
$$

where

$$
\mu_{I}=\frac{3}{2}+\lambda_{I}
$$

This is the same form for the equation of motion as in the single field case [5] except that the $\mu_{I}$ depend on slow-roll parameters relating to both the original fields $\phi_{1}$ and $\phi_{2}$.

Working at first order in slow roll, so that we can treat the slow-roll parameters and hence $\mu_{I}$ as constant, the solution is

$$
v_{I}=\frac{\sqrt{\pi}}{2} e^{i\left(\mu_{I}+1 / 2\right) \pi / 2}(-\tau)^{1 / 2} H_{\mu_{I}}^{(1)}(-k \tau) e_{I}(k)
$$

where $H_{\mu_{I}}^{(1)}(x)$ is a Hankel function of the first kind of order $\mu_{I}$ and the $e_{I}(k)$ are independent unit Gaussian random variables [9] satisfying

$$
\left\langle e_{I}(\mathbf{k})\right\rangle=0, \quad\left\langle e_{I}(\mathbf{k}) e_{J}^{*}\left(\mathbf{k}^{\prime}\right)\right\rangle=\delta_{I J} \delta^{3}\left(\mathbf{k}-\mathbf{k}^{\prime}\right) .
$$

The constant in front of the Hankel function solution for $v_{I}$ comes from imposing the early-time boundary condition

$$
v_{I} \longrightarrow \frac{1}{\sqrt{2 k}} e^{-i k \tau} e_{I}(k) \quad \text { as } \quad-k \tau \longrightarrow \infty .
$$

The corresponding late-time behavior $(k \tau \rightarrow 0)$ is

$$
\begin{aligned}
& v_{I} \longrightarrow \quad e^{i\left(\mu_{I}-1 / 2\right) \pi / 2} 2^{\mu_{I}-3 / 2} \frac{\Gamma\left(\mu_{I}\right)}{\Gamma(3 / 2)} \frac{1}{\sqrt{2 k}}(-k \tau)^{-1-\lambda_{I}} e_{I}(k) \\
& \simeq \quad i e^{i \lambda_{I} \pi / 2}\left(1+C \lambda_{I}\right) \frac{1}{\sqrt{2 k}}(-k \tau)^{-1-\lambda_{I}} e_{I}(k)
\end{aligned}
$$

where $C=2-\log 2-\gamma \approx 0.7296$ and $\gamma \approx 0.5772$ is the Euler-Mascheroni constant. Note however that although the change in first-order slow-roll parameters and hence $\mu_{I}$ in one Hubble time is second order in slow roll, see appendix $\mathrm{B}$, the time variation of the slow-roll parameters cannot be neglected over many Hubble times. Thus we can only reliably use the late-time solution (18) for a few Hubble times after Hubble-exit $\left(k=a_{*} H_{*}\right)$. We need to perform another rotation in field space to accurately track the evolution of the field perturbations over many expansion times on super-Hubble scales. 


\section{The adiabatic and isocurvature perturbations}

To follow the coupled evolution of the perturbations on large scales $(k \ll a H)$ it is more useful to rotate the field basis into adiabatic and entropy perturbations, $\delta \sigma$ and $\delta s$. Following Ref. [9], the local rotation is given by

$$
\left(\begin{array}{c}
\delta \sigma \\
\delta s
\end{array}\right)=S^{\dagger}\left(\begin{array}{c}
\delta \phi_{1} \\
\delta \phi_{2}
\end{array}\right) \quad \text { where } \quad S=\left(\begin{array}{cc}
\cos \theta & -\sin \theta \\
\sin \theta & \cos \theta
\end{array}\right)
$$

and the local rotation angle $\theta$, which is now a function of time, is given by

$$
\tan \theta=\frac{\dot{\phi}_{2}}{\dot{\phi}_{1}}
$$

The adiabatic and isocurvature perturbations are then related to the decoupled perturbations $v_{I}$ by

$$
\left(\begin{array}{c}
a \delta \sigma \\
a \delta s
\end{array}\right)=\left(\begin{array}{cc}
\cos (\Theta-\theta) & -\sin (\Theta-\theta) \\
\sin (\Theta-\theta) & \cos (\Theta-\theta)
\end{array}\right)\left(\begin{array}{c}
v_{1} \\
v_{2}
\end{array}\right)
$$

The eigenvalues of $U$, given in Eq. (11) can be written more compactly in terms of the $\sigma$ and $s$ slow-roll parameters (see appendix A)

$$
\lambda_{1,2} \simeq \frac{1}{2}\left[4 \epsilon-\left(\eta_{\sigma \sigma}+\eta_{s s}\right) \pm \sqrt{\varpi^{2}+4 \eta_{\sigma s}^{2}}\right]_{*}
$$

where

$$
\varpi=2 \epsilon-\left(\eta_{\sigma \sigma}-\eta_{s s}\right),
$$

and the global rotation angle $\Theta$ defined in Eq. (9) simplifies to

$$
\tan 2 \Theta=\left[\frac{\varpi \sin 2 \theta-2 \eta_{\sigma s} \cos 2 \theta}{\varpi \cos 2 \theta+2 \eta_{\sigma s} \sin 2 \theta}\right]_{*} .
$$

From the above equation it is easy to see that the two rotations, by $\Theta$ and $\theta$, are the same if and only if $\eta_{\sigma s}=0$, i.e. in the special case of a straight background trajectory since $[3] \dot{\theta} \simeq-H \eta_{\sigma s}$.

Since $v_{1}$ and $v_{2}$ are uncorrelated we have

$$
\begin{aligned}
a^{2}\left\langle|\delta \sigma|^{2}\right\rangle & =\frac{1}{2}\left(\left\langle v_{1}^{2}\right\rangle+\left\langle v_{2}^{2}\right\rangle\right)+\frac{1}{2} \cos 2(\Theta-\theta)\left(\left\langle v_{1}^{2}\right\rangle-\left\langle v_{2}^{2}\right\rangle\right), \\
a^{2}\left\langle\delta \sigma \delta s^{*}\right\rangle & =\frac{1}{2} \sin 2(\Theta-\theta)\left(\left\langle v_{1}^{2}\right\rangle-\left\langle v_{2}^{2}\right\rangle\right), \\
a^{2}\left\langle|\delta s|^{2}\right\rangle & =\frac{1}{2}\left(\left\langle v_{1}^{2}\right\rangle+\left\langle v_{2}^{2}\right\rangle\right)-\frac{1}{2} \cos 2(\Theta-\theta)\left(\left\langle v_{1}^{2}\right\rangle-\left\langle v_{2}^{2}\right\rangle\right),
\end{aligned}
$$

and from (20) and (24) the trigonometric terms can be written in terms of the slow-roll parameters

$$
\cos 2(\Theta-\theta)=\frac{\varpi}{\sqrt{\varpi^{2}+4 \eta_{\sigma s}^{2}}}+\mathcal{O}\left(\epsilon, \eta_{\sigma \sigma}, \eta_{\sigma s}, \eta_{s s}\right), \quad \sin 2(\Theta-\theta)=\frac{-2 \eta_{\sigma s}}{\sqrt{\varpi^{2}+4 \eta_{\sigma s}^{2}}}+\mathcal{O}\left(\epsilon, \eta_{\sigma \sigma}, \eta_{\sigma s}, \eta_{s s}\right) .
$$

A key observation is that $\delta \sigma$ and $\delta s$ are correlated at Hubble exit at first order in slow roll if the background trajectory is curved. Using the above relationships and Eq. (18) we can calculate the power spectra and cross-correlation at Hubble exit

$$
\begin{aligned}
& \mathcal{P}_{\sigma *}(k) \simeq\left(\frac{H_{*}}{2 \pi}\right)^{2}\left(1+(-2+6 C) \epsilon-2 C \eta_{\sigma \sigma}\right), \\
& \mathcal{C}_{\sigma s *}(k) \simeq-2 C \eta_{\sigma s}\left(\frac{H_{*}}{2 \pi}\right)^{2}, \\
& \mathcal{P}_{s *}(k) \simeq\left(\frac{H_{*}}{2 \pi}\right)^{2}\left(1+(-2+2 C) \epsilon-2 C \eta_{s s}\right),
\end{aligned}
$$

where here, and in the following, the slow-roll parameters are to be evaluated at Hubble exit, and we define the autocorrelation $\mathcal{P}_{x} \equiv \mathcal{C}_{x x}$ and the cross-correlation

$$
\mathcal{C}_{x y} \delta^{3}\left(\mathbf{k}-\mathbf{k}^{\prime}\right) \equiv \frac{4 \pi k^{3}}{(2 \pi)^{3}}\left\langle x(\mathbf{k}) y^{*}\left(\mathbf{k}^{\prime}\right)\right\rangle
$$




\section{OBSERVABLES AND FINAL POWER SPECTRA}

The comoving curvature perturbation during inflation is given in terms of the adiabatic field perturbations in the spatially flat gauge, by [9]

$$
\mathcal{R}=\frac{H}{\dot{\sigma}} \delta \sigma .
$$

Similarly a dimensionless isocurvature perturbation during inflation can be defined as [2]

$$
\mathcal{S}=\frac{H}{\dot{\sigma}} \delta s .
$$

A convenient dimensionless measure of the correlation angle $\Delta$ is

$$
\cos \Delta \equiv \frac{\mathcal{C}_{\mathcal{R S}}}{\mathcal{P}_{\mathcal{R}}^{1 / 2} \mathcal{P}_{\mathcal{S}}^{1 / 2}}
$$

It is straightforward to convert the power spectra and cross-correlation at Hubble exit, given in Eqs. (29-31), to the dimensionless variables

$$
\begin{aligned}
\mathcal{P}_{\mathcal{R} *} & \simeq\left(\frac{H_{*}^{2}}{\dot{\sigma}_{*} 2 \pi}\right)^{2}\left(1+(-2+6 C) \epsilon-2 C \eta_{\sigma \sigma}\right)=\mathcal{P}_{*}^{(0)}\left(1+a_{1}\right), \\
\mathcal{C}_{\mathcal{R} \mathcal{S}_{*}} & \simeq-2 C \eta_{\sigma s}\left(\frac{H_{*}^{2}}{\dot{\sigma}_{*} 2 \pi}\right)^{2}=\mathcal{P}_{*}^{(0)} a_{2} \\
\mathcal{P}_{\mathcal{S} *} & \simeq\left(\frac{H_{*}^{2}}{\dot{\sigma}_{*} 2 \pi}\right)^{2}\left(1+(-2+2 C) \epsilon-2 C \eta_{s s}\right)=\mathcal{P}_{*}^{(0)}\left(1+a_{3}\right), \\
\cos \Delta_{*} & \simeq-2 C \eta_{\sigma s}
\end{aligned}
$$

where we have defined

$$
\mathcal{P}_{*}^{(0)} \equiv\left(\frac{H_{*}^{2}}{\dot{\sigma}_{*} 2 \pi}\right)^{2}=\left(\frac{H_{*}}{2 \pi}\right)^{2} \frac{16 \pi G}{\epsilon^{H}},
$$

and the first-order slow-roll corrections are given by

$$
a_{1}=-2 \epsilon+2 C\left(3 \epsilon-\eta_{\sigma \sigma}\right), \quad a_{2}=-2 C \eta_{\sigma s}, \quad a_{3}=-2 \epsilon+2 C\left(\epsilon-\eta_{s s}\right) .
$$

\section{A. Super-Hubble evolution}

In order to calculate the primordial curvature and isocurvature perturbations some time after inflation has ended, which can be constrained by observations today we need to model the evolution of $\mathcal{R}$ and $\mathcal{S}$ on large scales by introducing the transfer functions

$$
\left(\begin{array}{c}
\mathcal{R} \\
\mathcal{S}
\end{array}\right)=\left(\begin{array}{cc}
1 & T_{\mathcal{R S}} \\
0 & T_{\mathcal{S S}}
\end{array}\right)\left(\begin{array}{c}
\mathcal{R} \\
\mathcal{S}
\end{array}\right)_{*}
$$

$T_{\mathcal{R} \mathcal{R}}=1$ because in the absence of isocurvature modes the adiabatic perturbation is conserved and $T_{\mathcal{S} \mathcal{R}}=0$ because the adiabatic perturbation cannot act as a source to the isocurvature perturbation.

On the other hand, $T_{\mathcal{R} S}$ and $T_{\mathcal{S S}}$, which parameterise the effect of entropy perturbations during inflation upon the primordial curvature and isocurvature perturbations, depend upon the full evolution on super-Hubble scales, both during inflation, and afterwards. Quite generally, we have

$$
\dot{\mathcal{R}}=\alpha(t) H \mathcal{S}, \quad \dot{\mathcal{S}}=\beta(t) H \mathcal{S}
$$

and thus we can write [3]

$$
T_{\mathcal{S S}}\left(t_{*}, t\right)=\exp \left(\int_{t_{*}}^{t} \beta\left(t^{\prime}\right) H\left(t^{\prime}\right) d t^{\prime}\right), \quad T_{\mathcal{R S}}=\int_{t_{*}}^{t} \alpha\left(t^{\prime}\right) T_{\mathcal{S} \mathcal{S}}\left(t_{*}, t^{\prime}\right) H\left(t^{\prime}\right) d t^{\prime} .
$$


The transfer functions contain an implicit scale dependence, through the dependence of the Hubble-exit time, $t_{*}$, which varies with scale, $k=(a H)_{*}$. The time dependence, calculated using the Leibniz integral rule, is

$$
\frac{1}{H}{ }_{*} \frac{\partial T_{\mathcal{S S}}}{\partial t_{*}}=-\beta_{*} T_{\mathcal{S S}}, \quad \frac{1}{H_{*}} \frac{\partial T_{\mathcal{R S}}}{\partial t_{*}}=-\alpha_{*}-\beta_{*} T_{\mathcal{R S}} .
$$

To calculate the spectral indices of the primordial power spectra to second order in slow roll it is thus necessary to also calculate $\alpha_{*}$ and $\beta_{*}$ to second order.

On large scales the time derivative of the curvature perturbation is [19]

$$
\dot{\mathcal{R}}=2 \dot{\theta} \mathcal{S} .
$$

Differentiating Eq. (20) for $\tan \theta$ gives

$$
\dot{\theta}=H \sin \theta \cos \theta\left(\delta_{1}^{H}-\delta_{2}^{H}\right),
$$

where $\delta_{I}^{H}$ are slow-roll parameters defined in appendix A. Then using appendix A to write $\delta_{I}^{H}$ in terms of the potential slow-roll parameters we find

$$
\alpha_{*} \cong\left(-2+\frac{8}{3} \epsilon-\frac{2}{3} \eta_{\sigma \sigma}-\frac{2}{3} \eta_{s s}\right) \eta_{\sigma s}-\frac{2}{3} \xi_{\sigma \sigma s}^{2} .
$$

As can be easily seen from (46), $\alpha_{*}=0$ in the case of a straight background trajectory in field space, and the curvature perturbation in that case is constant during inflation on super-Hubble scales.

To calculate $\beta_{*}$ we start with the evolution equation of $\delta s[9]$

$$
\ddot{\delta s}+3 H \dot{\delta s}+\left(\frac{k^{2}}{a^{2}}+V_{s s}+3 \dot{\theta}^{2}\right) \delta s=\frac{\dot{\theta}}{\dot{\sigma}} \frac{k^{2}}{2 \pi G a^{2}} \Psi,
$$

where the Bardeen potential $\Psi \simeq 4 \pi G(\dot{\sigma} / H) \delta \sigma$ during slow-roll on large scales [9]. Therefore we can drop the term on the right hand side of $(49)$ as well as the $\left(k^{2} / a^{2}\right) \delta s$ term on large scales. Rewriting the equation in terms of $\mathcal{S}$ we then find

$$
\ddot{\mathcal{S}}+\left(3 H+\frac{\dot{\epsilon}^{H}}{\epsilon^{H}}\right) \dot{\mathcal{S}}+\left(V_{s s}+3 \dot{\theta}^{2}+\frac{3}{2} H \frac{\dot{\epsilon}^{H}}{\epsilon^{H}}+\frac{1}{2} \frac{\ddot{\epsilon}^{H}}{\epsilon^{H}}-\frac{1}{4}\left(\frac{\dot{\epsilon}^{H}}{\epsilon^{H}}\right)^{2}\right) \mathcal{S}=0,
$$

so, to lowest order in slow roll, we have

$$
\dot{\mathcal{S}} \simeq H\left(-2 \epsilon+\eta_{\sigma \sigma}-\eta_{s s}\right) \mathcal{S} .
$$

We are able to calculate the next order corrections by taking the derivative of this and substituting it back into (50). After some calculation, we find

$$
\beta_{*} \cong \frac{1}{3}\left(-6 \epsilon+3 \eta_{\sigma \sigma}-3 \eta_{s s}+12 \epsilon^{2}+\eta_{\sigma \sigma}^{2}-\eta_{s s}^{2}-10 \epsilon \eta_{\sigma \sigma}+2 \epsilon \eta_{s s}+\xi_{\sigma \sigma \sigma}^{2}-\xi_{\sigma s s}^{2}\right) .
$$

\section{B. Final Power Spectra and spectral indices}

The primordial curvature perturbation, during the radiation-dominated era some time after inflation has ended, is given on large scales by

$$
\mathcal{R}=\psi+\frac{H \delta \rho}{\dot{\rho}} .
$$

It is this curvature perturbation that, for example, produces large-scale anisotropies in the cosmic microwave background. The conventional definition of the primordial isocurvature matter perturbation is given relative to the radiation density by

$$
\mathcal{S}=H\left(\frac{\delta \rho_{m}}{\dot{\rho}_{m}}-\frac{\delta \rho_{\gamma}}{\dot{\rho}_{\gamma}}\right)
$$


From the definition of the power spectra (32) and the transfer functions (42) it follows that

$$
\begin{aligned}
\mathcal{P}_{\mathcal{R}} & \simeq \mathcal{P}_{\mathcal{R} *}+2 T_{\mathcal{R S}} \mathcal{C}_{\mathcal{R S} *}+T_{\mathcal{R} \mathcal{S}}^{2} \mathcal{P}_{\mathcal{S} *} \\
\mathcal{C}_{\mathcal{R S}} & \simeq T_{\mathcal{S S}} \mathcal{C}_{\mathcal{R S} *}+T_{\mathcal{S}} T_{\mathcal{R S}} \mathcal{P}_{\mathcal{S} *} \\
\mathcal{P}_{\mathcal{S}} & \simeq T_{\mathcal{S} \mathcal{S}}^{2} \mathcal{P}_{\mathcal{S} *}
\end{aligned}
$$

and substituting in the power spectra at Hubble exit (36-38) we see that the primordial power spectra are

$$
\begin{aligned}
\mathcal{P}_{\mathcal{R}} & \simeq \mathcal{P}_{*}^{(0)}\left(1+T_{\mathcal{R S}}^{2}+a_{1}+2 T_{\mathcal{R S}} a_{2}+T_{\mathcal{R} \mathcal{S}}^{2} a_{3}\right) \\
\mathcal{C}_{\mathcal{R S}} & \simeq \mathcal{P}_{*}^{(0)} T_{\mathcal{S} \mathcal{S}}\left(T_{\mathcal{R S}}+a_{2}+T_{\mathcal{R S}} a_{3}\right) \\
\mathcal{P}_{\mathcal{S}} & \simeq \mathcal{P}_{*}^{(0)} T_{\mathcal{S} \mathcal{S}}^{2}\left(1+a_{3}\right)
\end{aligned}
$$

where $a_{1}, a_{2}$ and $a_{3}$ are the corrections from $\mathcal{P}_{*}^{(0)}$ for the initial power spectra as defined in Eqs. (40) and (41).

Note that the next-to-leading order corrections to the power spectra $(58-60)$ can be related to the tilts by the relation given in Ref. [4]

$$
\mathcal{P}_{X} \simeq \mathcal{P}_{X}^{(0)}\left(1+n_{T}-C n_{X}\right)
$$

where the subscript $X$ means that that the relation is true for the adiabatic, isocurvature and tensor power spectra and the cross correlation, and $\mathcal{P}_{X}^{(0)}$ is the power spectra at lowest order. The tilt of the gravitational wave power spectrum, $n_{T}$, is defined in section III D

The scale dependence depends on both the initial power spectra and the transfer functions, we can replace the dependence on the transfer functions with the observable correlation angle $\cos \Delta$, which at lowest order satisfies

$$
\cos \Delta^{(0)}=\frac{T_{\mathcal{R S}}}{\sqrt{1+T_{\mathcal{R S}}^{2}}}
$$

For details of the calculation and definitions of $\cos \Delta, \sin \Delta, \tan \Delta$ see appendix $\mathrm{C}$.

The final scalar tilts, defined as $n_{X}=d \ln \mathcal{P}_{X} / d \ln k$, up to second order in slow-roll parameters are ${ }^{1}$

$$
\begin{aligned}
n_{\mathcal{R}} \cong & -\left(6-4 \cos ^{2} \Delta\right) \epsilon+2 \sin ^{2} \Delta \eta_{\sigma \sigma}+4 \sin \Delta \cos \Delta \eta_{\sigma s}+2 \cos ^{2} \Delta \eta_{s s} \\
& +\left(-\frac{10}{3}-4 \cos ^{2} \Delta+C\left(24-16 \cos ^{2} \Delta\right)\right) \epsilon^{2}+\frac{2}{3} \sin ^{2} \Delta \eta_{\sigma \sigma}^{2}+\left(\frac{2}{3} \cos ^{2} \Delta+4 C\left(1-2 \cos ^{2} \Delta\right)\right) \eta_{\sigma s}^{2} \\
& +\frac{2}{3} \cos ^{2} \Delta \eta_{s s}^{2}+\left(-2+\frac{14}{3} \cos ^{2} \Delta+C\left(-16+12 \cos ^{2} \Delta\right)\right) \epsilon \eta_{\sigma \sigma}-\frac{4}{3} \sin \Delta \cos \Delta(1+6 C) \epsilon \eta_{\sigma s} \\
& -\frac{2}{3} \cos ^{2} \Delta(-1+6 C) \epsilon \eta_{s s}+\frac{4}{3} \sin \Delta \cos \Delta(1-3 C) \eta_{\sigma \sigma} \eta_{\sigma s}+\frac{4}{3} \sin \Delta \cos \Delta(1+3 C) \eta_{\sigma s} \eta_{s s} \\
& +\frac{2}{3} \sin ^{2} \Delta(1+3 C) \xi_{\sigma \sigma \sigma}^{2}+\frac{4}{3} \sin \Delta \cos \Delta(1+3 C) \xi_{\sigma \sigma s}^{2}+\frac{2}{3} \cos ^{2} \Delta(1+3 C) \xi_{\sigma s s}^{2}, \\
n_{\mathcal{C}} \cong & -2 \epsilon+2 \tan \Delta \eta_{\sigma s}+2 \eta_{s s} \\
& +\left(-\frac{22}{3}+8 C\right) \epsilon^{2}+\frac{2}{3}(1-6 C) \eta_{\sigma s}^{2}+\frac{2}{3} \eta_{s s}^{2}+\frac{8}{3}\left(1-\frac{3}{2} C\right) \epsilon \eta_{\sigma \sigma}-\frac{2}{3}(1+6 C) \tan \Delta \epsilon \eta_{\sigma s}+\frac{2}{3}(1-6 C) \epsilon \eta_{s s} \\
& +\frac{2}{3}(1-3 C) \tan \Delta \eta_{\sigma \sigma} \eta_{\sigma s}+\frac{2}{3}(1+3 C) \tan \Delta \eta_{\sigma s} \eta_{s s}+\frac{2}{3}(1+3 C) \tan \Delta \xi_{\sigma \sigma s}^{2}+\frac{2}{3}(1+3 C) \xi_{\sigma s s}^{2}, \\
n_{\mathcal{S}} \cong & \left.-2 \epsilon+2 \eta_{s s}(6) \frac{2}{3}+8 C\right) \epsilon^{2}+\frac{2}{3}(1-6 C) \eta_{\sigma s}^{2}+\frac{2}{3} \eta_{s s}^{2}+\frac{8}{3}\left(1-\frac{3}{2} C\right) \epsilon \eta_{\sigma \sigma}+\frac{2}{3}(1-6 C) \epsilon \eta_{s s}+\frac{2}{3}(1+3 C) \xi_{\sigma s s}^{2} .
\end{aligned}
$$

The running of the spectral index is defined as $\alpha_{X} \equiv d n_{X} / d \ln k$. Assuming that the power law approximation for $\mathcal{P}_{\mathcal{R}}, \mathcal{C}_{\mathcal{R} S}$ and $\mathcal{P}_{\mathcal{S}}$ are valid, the running will be second order in the slow roll approximation because the time derivatives

\footnotetext{
${ }^{1}$ In this notation a scale-invariant (Harrison-Zel'dovich) spectrum corresponds to $n_{\mathcal{R}}=0$.
} 
(or equivelantly $\ln k$ derivatives) of the first-order slow-roll parameters are second order. To leading order in slow roll (see also [4]),

$$
\begin{aligned}
\alpha_{\mathcal{R}} \cong & 8\left(-3+4 \cos ^{2} \Delta-2 \cos ^{4} \Delta\right) \epsilon^{2}+4 \sin ^{2} \Delta \cos ^{2} \Delta \eta_{\sigma \sigma}^{2}+4\left(1-4 \sin ^{2} \Delta \cos ^{2} \Delta\right) \eta_{\sigma s}^{2}+4 \sin ^{2} \Delta \cos ^{2} \Delta \eta_{s s}^{2} \\
& +4\left(4-7 \cos ^{2} \Delta+4 \cos ^{4} \Delta\right) \epsilon \eta_{\sigma \sigma}+32 \sin ^{3} \Delta \cos \Delta \epsilon \eta_{\sigma s}+4 \cos ^{2} \Delta\left(5-4 \cos ^{2} \Delta\right) \epsilon \eta_{s s} \\
& -8 \sin \Delta \cos \Delta\left(1-2 \cos ^{2} \Delta\right) \eta_{\sigma \sigma} \eta_{\sigma s}-8 \sin ^{2} \Delta \cos ^{2} \Delta \eta_{\sigma \sigma} \eta_{s s}+8 \sin \Delta \cos \Delta\left(1-2 \cos ^{2} \Delta\right) \eta_{\sigma s} \eta_{s s} \\
& -2 \sin ^{2} \Delta \xi_{\sigma \sigma \sigma}^{2}-4 \sin \Delta \cos \Delta \xi_{\sigma \sigma s}^{2}-2 \cos ^{2} \Delta \xi_{\sigma s s}^{2}, \\
\alpha_{C} \cong & -8 \epsilon^{2}+4\left(1-\tan ^{2} \Delta\right) \eta_{\sigma s}^{2}+4 \epsilon \eta_{\sigma \sigma}+4 \epsilon \eta_{s s}+4 \tan \Delta \eta_{\sigma \sigma} \eta_{\sigma s}-4 \tan \Delta \eta_{\sigma s} \eta_{s s}-2 \tan \Delta \xi_{\sigma \sigma s}^{2}-2 \xi_{\sigma s s}^{2} \\
\alpha_{\mathcal{S}} \cong & -8 \epsilon^{2}+4 \eta_{\sigma s}^{2}+4 \epsilon \eta_{\sigma \sigma}+4 \epsilon \eta_{s s}-2 \xi_{\sigma s s}^{2} .
\end{aligned}
$$

It would be a straightforward but long calculation to include the next to leading terms also, since all that is required is to differentiate the tilts including the leading corrections as given above.

The spectral indices of $\mathcal{P}_{\mathcal{R}}$ and $\mathcal{P}_{\mathcal{S}}$ are both slow roll suppressed while generically $\alpha_{\mathcal{R} / \mathcal{S}} \sim \mathcal{O}\left(n_{\mathcal{R} / \mathcal{S}}^{2}\right)$ so the power spectra are both weakly scale dependent and well approximated by power laws. However the cross-correlation can be strongly scale-dependent if $\tan \Delta$ is large, specifically if

$$
\tan \Delta \eta_{\sigma s} \sim \mathcal{O}(1)
$$

In this case the running $\alpha_{C} \sim \mathcal{O}\left(\left(\tan \Delta \eta_{\sigma s}\right)^{2}\right)$ is also large and so $\mathcal{C}_{\mathcal{R} S}$ is not well approximated by a power law. The problem is that $T_{\mathcal{R S}}$ does not have a power law shape whenever $\left|\alpha_{*}\right| \gtrsim\left|\beta_{*} T_{\mathcal{R S}}\right|$ as shown by (45) and then $\mathcal{C}_{\mathcal{R S}}$ will not be close to power law either $(56)$. We can see in this case $C_{\mathcal{R S}}$ has an approximate log-dependence on wavenumber from (45) and (59). Fortunately this problem only occurs when $\cos \Delta \approx 0$, i.e. the perturbations are nearly uncorrelated and then $n_{\mathcal{C}}$ will be nearly unconstrained by observations. Because $T_{\mathcal{R} S}$ has to be small when it is not a power law (recalling that $\tan \Delta \sim 1 / T_{\mathcal{R} S}$ ), $\mathcal{P}_{\mathcal{R}}$ remains a power law in spite of its leading order dependence on $T_{\mathcal{R} \mathcal{S}}^{2}(55)$. We can see this more explicitly by parameterizing $\mathcal{P}_{\mathcal{R}}$ as the sum of two power laws which we discuss next.

\section{Alternative parametrisation of the power spectra}

A different way to write the primordial power spectra is to split the adiabatic power spectrum into a part generated by the inflationary adiabatic perturbations and a second adiabatic perturbation generated from the inflationary entropy perturbation [20], (see also [2, 21]),

$$
\begin{aligned}
\mathcal{P}_{\mathcal{R}} & =A_{r}^{2}\left(\frac{k}{k_{0}}\right)^{n_{\mathrm{ad} 1}}+A_{s}^{2}\left(\frac{k}{k_{0}}\right)^{n_{\mathrm{ad} 2}}, \\
\mathcal{C}_{\mathcal{R} \mathcal{S}} & =A_{s} B\left(\frac{k}{k_{0}}\right)^{n_{\mathrm{cor}}}, \\
\mathcal{P}_{\mathcal{S}} & =B^{2}\left(\frac{k}{k_{0}}\right)^{n_{\mathrm{iso}}},
\end{aligned}
$$

where $k_{0}$ is the pivot scale. The amplitude of the primordial curvature perturbation spectra are given in terms of the power spectra at Hubble exit and the primordial transfer functions (see Eq. (55)) by

$$
A_{r}^{2}=\left[\mathcal{P}_{\mathcal{R} *}\right]_{k_{0}}, \quad A_{s}^{2}=\left[T_{\mathcal{R} \mathcal{S}}^{2} \mathcal{P}_{\mathcal{S} *}+2 T_{\mathcal{R S}} \mathcal{C}_{\mathcal{R} \mathcal{S} *}\right]_{k_{0}}
$$

$A_{s}^{2}$ and $A_{r}^{2}$ can also be simply written in terms of the correlation angle, (35),

$$
A_{r}^{2}=\left[\mathcal{P}_{\mathcal{R}} \sin ^{2} \Delta\right]_{k_{0}}, \quad A_{s}^{2}=\left[\mathcal{P}_{\mathcal{R}} \cos ^{2} \Delta\right]_{k_{0}}
$$

Only 3 of the four tilts are independent because $n_{\text {cor }}=\left(n_{\text {ad2 } 2}+n_{\text {iso }}\right) / 2 . \mathcal{C}_{\mathcal{R} \mathcal{S}}$ and $\mathcal{P}_{\mathcal{S}}$ are the same as in the standard notation so $n_{\text {cor }}=n_{\mathcal{C}}, n_{\text {iso }}=n_{\mathcal{S}}$ and $n_{\text {ad2 }}=2 n_{\mathcal{C}}-n_{\mathcal{S}} . n_{\text {ad1 }}$ is the tilt of the adiabatic perturbations at the Hubble-exit 
time of $k_{0}$. The four spectral indices are therefore

$$
\begin{aligned}
n_{\mathrm{ad} 1} \cong & -6 \epsilon+2 \eta_{\sigma \sigma}+\left(-\frac{10}{3}+24 C\right) \epsilon^{2}+\frac{2}{3} \eta_{\sigma \sigma}^{2}+\frac{2}{3}(1+6 C) \eta_{\sigma s}^{2}-(2+16 C) \epsilon \eta_{\sigma \sigma}+\left(\frac{2}{3}+2 C\right) \xi_{\sigma \sigma \sigma}^{2}, \\
n_{\mathrm{ad} 2} \cong & -2 \epsilon+4 \tan \Delta \eta_{\sigma s}+2 \eta_{s s}+\left(-\frac{22}{3}+8 C\right) \epsilon^{2}+\frac{2}{3}(1-6 C) \eta_{\sigma s}^{2}+\frac{2}{3} \eta_{s s}^{2}+\left(\frac{8}{3}-4 C\right) \epsilon \eta_{\sigma \sigma} \\
& -\left(\frac{4}{3}+8 C\right) \tan \Delta \epsilon \eta_{\sigma s}+\left(\frac{2}{3}-4 C\right) \epsilon \eta_{s s}+\frac{4}{3}(1-3 C) \tan \Delta \eta_{\sigma \sigma} \eta_{\sigma s}+\frac{4}{3}(1+3 C) \tan \Delta \eta_{\sigma s} \eta_{s s} \\
& +\frac{4}{3}(1+3 C) \tan \Delta \xi_{\sigma \sigma s}^{2}+\frac{2}{3}(1+3 C) \xi_{\sigma s s}^{2},
\end{aligned}
$$

while $n_{\text {cor }}=n_{\mathcal{C}}$ and $n_{\text {iso }}=n_{\mathcal{S}}$ are written explicitly in (64) and (65) respectively.

In the case when $\tan \Delta \eta_{\sigma s}$ is large $n_{\mathrm{ad} 2}$ is large and so is $\alpha_{\mathrm{nad}_{2}}=2 \alpha_{\mathcal{C}}-\alpha_{\mathcal{S}}$. Hence the second term of the primordial adiabatic power spectrum (70) is not well parameterised by power law. However $\mathcal{P}_{\mathcal{R}}$ can still be well approximated by a single power law because $A_{s}^{2} \ll A_{r}^{2}$ from (74) and the requirement that $|\tan \Delta| \gg 1$.

\section{Gravitational Waves}

Scalar and tensor perturbations are decoupled, so the gravitational wave power spectrum is the same as in the single-field result, and the amplitude of gravitational waves remains frozen-in on large scales after Hubble exit during inflation, [5],

$$
\mathcal{P}_{T}=\mathcal{P}_{T *} \simeq 64 \pi G\left(\frac{H_{*}}{2 \pi}\right)^{2}(1+2(-1+C) \epsilon) .
$$

The tilt and running at second order in slow roll can easily be calculated from this power spectrum to give

$$
\begin{aligned}
& n_{T} \cong-2 \epsilon\left[1+\left(\frac{11}{3}-4 C\right) \epsilon+\left(-\frac{4}{3}+2 C\right) \eta_{\sigma \sigma}\right], \\
& \alpha_{T} \cong-8 \epsilon^{2}+4 \epsilon \eta_{\sigma \sigma} .
\end{aligned}
$$

The tensor-scalar ratio ${ }^{2}$ at Hubble exit is the same as in the single-field case

$$
r_{*} \equiv \frac{\mathcal{P}_{\mathcal{R} *}}{\mathcal{P}_{T *}} \cong 16 \epsilon\left[1-\left(\frac{4}{3}+4 C\right) \epsilon+\left(\frac{2}{3}+2 C\right) \eta_{\sigma \sigma}\right],
$$

However some time after Hubble exit the scalar curvature perturbation may have evolved due to the effect of nonadiabatic perturbations. From the definition (35), the final power spectra (58-60) and the curvature power spectrum at Hubble exit (36) it follows that

$$
\mathcal{P}_{\mathcal{R} *} \cong \mathcal{P}_{\mathcal{R}} \sin ^{2} \Delta
$$

and thus in the radiation-dominated era we have

$$
r \cong 16 \epsilon \sin ^{2} \Delta\left[1-\left(\frac{4}{3}+4 C\right) \epsilon+\left(\frac{2}{3}+2 C\right) \eta_{\sigma \sigma}\right] .
$$

Note that the only difference from the single field tensor-scalar relation is the addition of the $\sin ^{2} \Delta$ factor, this explains why the observational upper bound on $r$ does not provide a direct upper bound on $\epsilon$ in the multiple field case.

\footnotetext{
2 Note that there are various definitions for the tensor-scalar ratio, we use the definition which satisfies $r \simeq 16 \epsilon$ to first order at Hubble exit as used in [21], for example.
} 


\section{MODEL DEPENDENT RELATIONS}

\section{A. Straight background trajectory}

In the case of a straight background trajectory in field space (during the time of Hubble exit for observable modes) the calculation simplifies considerably since then $\dot{\theta}=0$. From (48) this requires that the slow-roll parameters $\eta_{\sigma s}=\xi_{\sigma \sigma s}^{2}=0$. In the case of a symmetric potential [11] the background trajectory will be straight since any orthogonal velocities will decay quickly. A straight background trajectory is also the attractor solution of some assisted inflation models [13-15]. The inflaton and curvaton scenarios are two classes of inflation models with a straight background trajectory which we discuss in the following two subsections.

There is a consistency relation independent of the gravitational wave background which holds whenever the background trajectory is straight and which is valid at all orders in slow roll. From (49) the adiabatic and entropy perturbations decouple exactly when $\dot{\theta}=0$. Therefore the rotations performed before and after Hubble exit are the same, $\Theta=\theta$, as was already shown to lowest order in slow roll from (24) and the adiabatic and isocurvature perturbations are uncorrelated at Hubble exit. Equations (56) and (57) with $\mathcal{C}_{\mathcal{R S} *}=0$ imply

$$
T_{\mathcal{S S}} \mathcal{C}_{\mathcal{R S}}=T_{\mathcal{R S}} \mathcal{P}_{\mathcal{S}}
$$

From (46) it follows that $\alpha_{*}=0$ and therefore from (45), $T_{\mathcal{S S}}$ and $T_{\mathcal{R S}}$ have exactly the same $k$ dependence. Taking the derivatives of (83) with respect to wavenumber shows that

$$
\begin{aligned}
n_{\mathcal{C}} & =n_{\mathcal{S}}, \\
\alpha_{\mathcal{C}} & =\alpha_{S},
\end{aligned}
$$

and these consistency relations are true to all orders in slow roll. We can check explicitly that it holds up to second order in slow roll from Eqs. (64) and (65).

The tilts (63) and (65) simplify somewhat to give

$$
\begin{aligned}
n_{\mathcal{R}} \cong & -\left(6-4 \cos ^{2} \Delta\right) \epsilon+2 \sin ^{2} \Delta \eta_{\sigma \sigma}+2 \cos ^{2} \Delta \eta_{s s}+\left(-\frac{10}{3}-4 \cos ^{2} \Delta+C\left(24-16 \cos ^{2} \Delta\right)\right) \epsilon^{2} \\
& +\frac{2}{3} \sin ^{2} \Delta \eta_{\sigma \sigma}^{2}+\frac{2}{3} \cos ^{2} \Delta \eta_{s s}^{2}+\left(-2+\frac{14}{3} \cos ^{2} \Delta+C\left(-16+12 \cos ^{2} \Delta\right)\right) \epsilon \eta_{\sigma \sigma}-\frac{2}{3} \cos ^{2} \Delta(-1+6 C) \epsilon \eta_{s s} \\
& +\frac{2}{3}(1+3 C) \sin ^{2} \Delta \xi_{\sigma \sigma \sigma}^{2}+\frac{2}{3}(1+3 C) \cos ^{2} \Delta \xi_{\sigma s s}^{2}, \\
n_{\mathcal{S}} \cong & -2 \epsilon+2 \eta_{s s}+\left(-\frac{22}{3}+8 C\right) \epsilon^{2}+\frac{2}{3} \eta_{s s}^{2}+\left(\frac{8}{3}-4 C\right) \epsilon \eta_{\sigma \sigma}+\left(\frac{2}{3}-4 C\right) \epsilon \eta_{s s}+\frac{2}{3}(1+3 C) \cos ^{2} \Delta \xi_{\sigma s s}^{2},
\end{aligned}
$$

and the running is given by

$$
\begin{aligned}
\alpha_{\mathcal{R}} \cong & 8\left(-3+4 \cos ^{2} \Delta-2 \cos ^{4} \Delta\right) \epsilon^{2}+4 \sin ^{2} \Delta \cos ^{2} \Delta \eta_{\sigma \sigma}^{2}+4 \sin ^{2} \Delta \cos ^{2} \Delta \eta_{s s}^{2}+4\left(4-7 \cos ^{2} \Delta+4 \cos ^{4} \Delta\right) \epsilon \eta_{\sigma \sigma} \\
& +4 \cos ^{2} \Delta\left(5-4 \cos ^{2} \Delta\right) \epsilon \eta_{s s}-8 \sin ^{2} \Delta \cos ^{2} \Delta \eta_{\sigma \sigma} \eta_{s s}-2 \sin ^{2} \Delta \xi_{\sigma \sigma \sigma}^{2}-2 \cos ^{2} \Delta \xi_{\sigma s s}^{2} \\
\alpha_{\mathcal{S}} \cong & -8 \epsilon^{2}+4 \epsilon \eta_{\sigma \sigma}+4 \epsilon \eta_{s s}-2 \xi_{\sigma s s}^{2} .
\end{aligned}
$$

\section{B. Inflaton scenario}

If the background trajectory is straight during inflation then this direction in field space, $\sigma$, can be identified as the inflaton field. Other fields orthogonal to the inflaton are time-independent along the background trajectory, but quantum fluctuations in light fields $\left(\eta_{s s}<1\right)$ do generate isocurvature perturbations during inflation. If we further assume that these isocurvature perturbations remain decoupled from the inflaton and radiation density during and after inflation then the curvature perturbations today are purely due to inflaton field perturbations, i.e., $T_{\mathcal{R S}}=0$. We refer to this as the inflaton scenario. Hence any isocurvature perturbations that survive into the radiation era are uncorrelated with the curvature perturbations, $\mathcal{C}_{\mathcal{R} \mathcal{S}}=0$ and $\cos \Delta=0$.

The tilts in the inflaton scenario reduce to

$$
\begin{aligned}
& n_{\mathcal{R}} \cong-6 \epsilon+2 \eta_{\sigma \sigma}+\left(-\frac{10}{3}+24 C\right) \epsilon^{2}+\frac{2}{3} \eta_{\sigma \sigma}^{2}-(2+16 C) \epsilon \eta_{\sigma \sigma}+\left(\frac{2}{3}+2 C\right) \xi_{\sigma \sigma \sigma}^{2} \\
& n_{\mathcal{S}} \cong-2 \epsilon+2 \eta_{s s}+\left(-\frac{22}{3}+8 C\right) \epsilon^{2}+\frac{2}{3} \eta_{s s}^{2}+\left(\frac{8}{3}-4 C\right) \epsilon \eta_{\sigma \sigma}+\left(\frac{2}{3}-4 C\right) \epsilon \eta_{s s}+\frac{2}{3}(1+3 C) \xi_{\sigma s s}^{2}
\end{aligned}
$$


and the running is given by

$$
\begin{aligned}
& \alpha_{\mathcal{R}} \cong-24 \epsilon^{2}+16 \epsilon \eta_{\sigma \sigma}-2 \xi_{\sigma \sigma \sigma}^{2} \\
& \alpha_{\mathcal{S}} \cong-8 \epsilon^{2}+4 \epsilon \eta_{\sigma \sigma}+4 \epsilon \eta_{s s}-2 \xi_{\sigma s s}^{2}
\end{aligned}
$$

The results for the curvature perturbation spectrum in the inflaton case is identical to the standard result for singlefield inflation [5]. Since the curvature perturbations are frozen in from Hubble exit in this case, and the gravitational waves also are frozen in, the tensor-scalar ratio is also unchanged from the single field case

$$
r \cong 16 \epsilon\left[1-\left(\frac{4}{3}+4 C\right) \epsilon+\left(\frac{2}{3}+2 C\right) \eta_{\sigma \sigma}\right],
$$

and the standard single field consistency relations apply, see [22]

$$
r \cong-8 n_{T}\left[1-\frac{1}{2} n_{T}+n_{\mathcal{R}}\right]
$$

Note that by differentiating this expression we can obtain an infinite hierarchy of consistency relations at higher order in the slow-roll parameters [22], the first of which is $[12,23]$

$$
\alpha_{T} \cong n_{T}\left(n_{T}-n_{\mathcal{R}}\right)
$$

\section{Curvaton scenario}

In the curvaton scenario [16] the primordial curvature perturbation during the radiation dominated era is generated from isocurvature field fluctuations in a curvaton field during inflation. These curvaton perturbations lead to an inhomogeneous radiation density after inflation if the curvaton is a weakly coupled, massive scalar field whose energy density is non-negligible when it finally decays into radiation some time after inflation.

If the effect of curvaton perturbations is assumed to dominate over the contribution from the inflaton, our chosen normalisation of the dimensionless isocurvature perturbations during inflation (34), implies that $\left|T_{\mathcal{R S}}\right| \gg 1$. Thus we have $\cos \Delta= \pm 1$ in Eq. (35). The scalar tilt (63) and running (66) are therefore

$$
\begin{aligned}
& n_{\mathcal{R}} \cong-2 \epsilon+2 \eta_{s s}+\left(-\frac{22}{3}+8 C\right) \epsilon^{2}+\frac{2}{3} \eta_{s s}^{2}+\left(\frac{8}{3}-4 C\right) \epsilon \eta_{\sigma \sigma}+\left(\frac{2}{3}-4 C\right) \epsilon \eta_{s s}+\frac{2}{3}(1+3 C) \xi_{\sigma s s}^{2} \\
& \alpha_{\mathcal{R}} \cong-8 \epsilon^{2}+4 \epsilon \eta_{\sigma \sigma}+4 \epsilon \eta_{s s}-2 \xi_{\sigma s s}^{2} .
\end{aligned}
$$

If the inflaton perturbation, i.e. the scalar perturbation at Hubble exit, is negligible, then from Eq. (80) so is the gravitational wave background. We see that for $\sin \Delta=0$ Eq. (82) implies $r=0$.

Isocurvature perturbations offer an alternative consistency relation in the curvaton scenario. Not only the primordial curvature perturbation but also any residual isocurvature perturbation [24] is due to the curvaton field fluctuations during inflation. Thus the primordial curvature and isocurvature perturbations are $100 \%$ correlated (or anti-correlated), and we must have

$$
\begin{aligned}
& n_{\mathcal{R}}=n_{C}=n_{\mathcal{S}}, \\
& \alpha_{\mathcal{R}}=\alpha_{C}=\alpha_{\mathcal{S}} .
\end{aligned}
$$

\section{Curvature perturbations from broken symmetries}

In [25] it was proposed that the curvature perturbation seen today may be due to the isocurvature perturbations during inflation in a nearly symmetric potential. The isocurvature perturbations are then converted into curvature perturbations during instant preheating at the end of inflation [26]. The key parameter of the preheating is the minimum distance to the minimum of the potential reached along the inflaton trajectory and perturbations of this minimum distance are due to the isocurvature perturbations.

The nearly symmetric 2-field potential considered in [25] is

$$
V\left(\phi_{1}, \phi_{2}\right)=\frac{m^{2}}{2}\left(\phi_{1}^{2}+\frac{\phi_{2}^{2}}{1+x}\right),
$$


where $x$ is the symmetry breaking parameter which is assumed to satisfy $0 \leq x \ll 1$. We will assume the symmetry is only weakly broken and so $x$ is of the same order as the slow roll parameters or less. Hence we will neglect terms of order $x \epsilon^{2}$ and $x^{2} \epsilon$.

The values of the slow-roll parameters depend on the angle of the background trajectory which is a free parameter of the theory. The distance from the minimum of the potential when observable scales today were leaving the horizon during inflation (i.e. about 60 efoldings before the end of inflation) is fixed at $\sigma=\sqrt{\phi_{1}^{2}+\phi_{2}^{2}} \simeq 3 M_{P l}$. The $\phi_{1}$ and $\phi_{2}$ field values can be related to the differential rotation angle (19), defined by (20), by

$$
\phi_{1} \simeq \sigma \cos \theta\left(1-x \sin ^{2} \theta\right), \quad \phi_{2} \simeq \sigma \sin \theta\left(1+x \cos ^{2} \theta\right) .
$$

These can be derived by applying slow-roll approximations to the equations of motion

$$
\ddot{\phi}_{1}+3 H \dot{\phi}_{1}+m^{2} \phi_{1}=0, \quad \ddot{\phi}_{2}+3 H \dot{\phi}_{2}+\frac{m^{2}}{1+x} \phi_{2}=0 .
$$

Note that in the case of a symmetric potential, $(x=0)$, the background trajectory is straight with a polar angle coinciding with $\theta[11]$.

The potential can be written as

$$
V \simeq \frac{m^{2}}{2} \sigma^{2}\left(1-x \sin ^{2} \theta\right)
$$

The slow-roll parameters are therefore

$$
\epsilon=\frac{1}{4 \pi G} \frac{1}{\sigma^{2}}, \quad \eta_{\sigma \sigma}=\epsilon, \quad \eta_{\sigma s} \simeq-x \epsilon \sin \theta \cos \theta, \quad \eta_{s s} \simeq \epsilon(1-x \cos 2 \theta), \quad \xi_{\sigma \sigma \sigma}^{2}=\xi_{\sigma \sigma s}^{2}=\xi_{\sigma s s}^{2}=0 .
$$

If we suppose that the isocurvature perturbation during inflation dominates the primordial curvature perturbation we have $\cos \Delta=1$ and the spectral tilt is

$$
n_{\mathcal{R}} \cong-2 x \epsilon \cos 2 \theta-\frac{10}{3} \epsilon^{2} .
$$

Note that it is quite possible for the second-order in slow-roll contribution to dominate over the first-order result. In the limiting case of a symmetric potential, $V=m^{2} \sigma^{2} / 2$, there is no contribution at first order and the tilt is $n_{\mathcal{R}}=-10 \epsilon^{2} / 3$ as calculated in [11] using another method.

\section{CONCLUSIONS}

We have calculated the primordial curvature and isocurvature perturbations for an arbitrary model of two field inflation including first-order corrections in the slow-roll expansion. We have calculated the power spectra and the cross-correlation, including the first order corrections [Eqs. (58), (59) and (60)] which allows us to calculate the spectral tilts to second order in slow roll [Eqs. (63), (64) and (65)]. We find as expected that the scale dependence of the curvature and isocurvature power spectra are small, being first order in slow roll. However the scale-dependence of the cross-correlation is not necessarily small. It becomes large for $\eta_{\sigma s}>\cos \Delta$, where the slow-roll parameter $\eta_{\sigma s}$, defined in appendix A, determines the curvature of the trajectory in field space. Similarly we find that the running of the curvature and isocurvature tilt is very small, second order in slow roll, and thus the scale-dependence is well-fit by a power-law. However the scale dependence of the cross-correlation is not well-described by a power-law if $\eta_{\sigma s}>\cos \Delta$.

We use the approach of Gordon et al. [9] to evolve the instantaneous adiabatic and entropy field perturbations on super-Hubble scales. This approach was generalized to an arbitrary number of fields in curved field space by Nibbelink and van Tent $[4,10]$, who also calculated the leading slow-roll corrections to the primordial curvature and isocurvature power spectra, making some assumptions about the evolution of the universe after inflation. The isocurvature perturbations can be evolved independently of the curvature perturbations on large scales, but the curvature perturbation can be altered by non-adiabatic perturbations. The primordial power spectra are thus dependent upon two transfer functions, $T_{\mathcal{R} S}$ and $T_{\mathcal{S S}}$, whose magnitude we leave arbitrary, but whose scale-dependence can be determined in terms of the slow-roll parameters at Hubble-exit during inflation [3].

However on small scales (at early times during inflation) the coupling between the instantaneous adiabatic and entropy modes can become large leaving the initial vacuum state ambiguous in terms of these variables. Instead we defined an orthonormal basis in field space in which the interaction between fields is negligible at early times and 
minimises the interaction for a given mode at Hubble-exit. In this basis the effective mass matrix is diagonalised and the field perturbations are uncorrelated at Hubble exit at first order in slow roll. But we find that the instantaneous adiabatic and entropy field perturbations of Gordon et al. [9] are correlated at Hubble exit at first order in slow roll, $\cos \Delta_{*} \propto \eta_{\sigma s}$, unless the trajectory in field space is a straight line. Correlations between perturbations during 2-field inflation was also studied in [18].

In $[3,27]$ it was shown that the well known single-field consistency relation $r \simeq-8 n_{T}$ can be generalized to the case of two-field inflation to give $r \simeq-8 n_{T} \sin ^{2} \Delta$. Including the next-order correction terms the two-field consistency relation becomes

$$
r \cong-8 n_{T} \sin ^{2} \Delta\left[1-\frac{1}{2} n_{T}+\frac{1}{\sin ^{2} \Delta} n_{\mathcal{R}}-2 \frac{\cos ^{2} \Delta}{\sin ^{2} \Delta} n_{\mathcal{C}}+\frac{\cos ^{2} \Delta}{\sin ^{2} \Delta} n_{\mathcal{S}}\right] .
$$

At leading order in slow roll with two fields, there are four slow-roll parameters $\left(\epsilon, \eta_{\sigma \sigma}, \eta_{\sigma s}\right.$ and $\left.\eta_{s s}\right)$, and two transfer functions $\left(T_{\mathcal{R} \mathcal{S}}\right.$ and $T_{\mathcal{S} \mathcal{S}}$ ) as well as the energy scale (or Hubble parameter) at Hubble exit during inflation. However there are, in principle, eight observables, corresponding to the primordial curvature and isocurvature power spectra, their cross-correlation spectrum, the tensor spectrum and the tilts of all four spectra. Thus there is one consistency relation [3]. At second-order we have three more slow-roll parameters $\left(\xi_{\sigma \sigma \sigma}^{2}, \xi_{\sigma \sigma s}^{2}\right.$ and $\left.\xi_{\sigma s s}^{2}\right)$. Although $\xi_{\text {sss }}^{2}$ is in general non-zero it does not appear in the calculation of the power spectra, because whenever we take a time derivative (or derivative with respect to scale) we are differentiating along the background trajectory, and therefore with respect to the adiabatic field, $\sigma$ and not $s$. But we also have four more observables at this order, corresponding to the running of the four spectra. And thus we have one more consistency relation. Of course, there is no guarantee that either the primordial isocurvature perturbations or gravitational waves will be large enough to be detected.

We can differentiate Eq. (107) with respect to wavenumber, to find a higher-order consistency relation in terms of the running of the tensor spectrum, see [4],

$$
\alpha_{T} \sin ^{2} \Delta \cong n_{T}\left[n_{T}-n_{\mathcal{R}}+\cos ^{2} \Delta\left(2 n_{\mathcal{C}}-n_{\mathcal{S}}-n_{T}\right)\right] .
$$

We could include the third-order correction to this consistency relation by including the second-order correction term in (107), but the result would be extremely long. Since (107) holds on all scales we could repeatedly differentiate it to calculate an infinite hierarchy of consistency relations, as done in the single field case [22].

In the case of totally correlated curvature and isocurvature perturbations (i.e. $\cos \Delta=0$ ) the first consistency relation (107) is trivially satisfied since both $r=0$ and $\sin \Delta=0$. This corresponds for example to the curvaton scenario discussed in section IV C. However in this case there is an alternative set of consistency relations, $n_{\mathcal{S}}=n_{\mathcal{C}}=$ $n_{\mathcal{R}}$ at first order and $\alpha_{\mathcal{S}}=\alpha_{\mathcal{C}}=\alpha_{\mathcal{R}}$ at second order and so on to give an alternative infinite hierarchy of relations.

Finally note that observational constraints on the tensor-scalar ratio in the single field case directly constrain $\epsilon$ to be small, and then the near scale invariance of the scalar tilt also constrains $\eta$ to be small. Hence higher-order slow-roll corrections in single field inflation are constrained to be very small. However in two-field inflation the upper bound on the tensor-scalar ratio does not provide a direct constrain on $\epsilon$, and the near scale invariance of the adiabatic power spectrum only constrains a certain combination of the slow-roll parameters to be small. Hence the higher-order slow-roll corrections in two-field inflation can be significant.

\section{Acknowledgments}

The authors are grateful to Jussi Väliviita for comments. The authors are grateful to Bartjan van Tent for drawing our attention to related results in [4] and pointing out a correction to Eq. (108) in v1 of this paper. The authors are grateful to Ki-Young Choi for pointing out missing terms in the slow roll derivatives, Eqs. (B2-B5) in v2 of this paper. This leads to correction at second order in slow roll to several equations, most importantly for the tilts $(63-65,75,76)$, and the runnings (66-68). CB acknowledges financial support from the EPSRC.

\section{APPENDIX A: THE SLOW-ROLL PARAMETERS}

Different papers use different definitions of the slow-roll parameters, some of which are equivalent at first order, but none are equivalent at second order. The only ones which we shall use are 
First order Hubble

$$
\begin{aligned}
\epsilon^{H} & =-\frac{\dot{H}}{H^{2}}=\frac{8 \pi G}{2} \frac{\dot{\sigma}^{2}}{H^{2}}=\epsilon_{11}^{H}+\epsilon_{22}^{H}, \\
\epsilon_{I J}^{H} & =\frac{8 \pi G}{2} \frac{\dot{\phi}_{I} \dot{\phi}_{J}}{H^{2}}, \\
\delta_{I}^{H} & =-\frac{\ddot{\phi}_{I}}{H \dot{\phi}_{I}} \\
\delta_{\sigma}^{H} & =-\frac{\ddot{\sigma}}{H \dot{\sigma}} .
\end{aligned}
$$

First order potential

$$
\begin{aligned}
& \epsilon=\frac{1}{16 \pi G}\left(\frac{V_{\sigma}}{V}\right)^{2}, \\
& \eta_{\sigma s}=\frac{1}{8 \pi G} \frac{V_{\sigma s}}{V}, \quad \eta_{\sigma \sigma}=\frac{1}{8 \pi G} \frac{V_{\sigma \sigma}}{V}, \quad \eta_{s s}=\frac{1}{8 \pi G} \frac{V_{s s}}{V} .
\end{aligned}
$$

Second order potential

$$
\begin{array}{ll}
\xi_{\sigma \sigma \sigma}^{2}=\frac{1}{(8 \pi G)^{2}} \frac{V_{\sigma \sigma \sigma} V_{\sigma}}{V^{2}}, & \xi_{\sigma \sigma s}^{2}=\frac{1}{(8 \pi G)^{2}} \frac{V_{\sigma \sigma s} V_{\sigma}}{V^{2}} \\
\xi_{\sigma s s}^{2}=\frac{1}{(8 \pi G)^{2}} \frac{V_{\sigma s s} V_{\sigma}}{V^{2}}, & \xi_{s s s}^{2}=\frac{1}{(8 \pi G)^{2}} \frac{V_{s s s} V_{\sigma}}{V^{2}}
\end{array}
$$

Note that the superscript 2 refers to the quantity being a second order slow-roll parameter.

Slow-roll parameters defined in terms of the Hubble parameter can be related to the potential slow-roll parameters by

$$
\begin{aligned}
\epsilon^{H} \cong \epsilon-\frac{4}{3} \epsilon^{2}+\frac{2}{3} \epsilon \eta_{\sigma \sigma} \\
\delta_{\sigma}^{H} \cong-\epsilon+\eta_{\sigma \sigma}+\frac{8}{3} \epsilon^{2}-\frac{8}{3} \epsilon \eta_{\sigma \sigma}+\frac{1}{3} \eta_{\sigma \sigma}^{2}+\frac{1}{3} \eta_{\sigma s}^{2}+\frac{1}{3} \xi_{\sigma \sigma \sigma}^{2} \\
\delta_{I}^{H} \cong-\epsilon^{H}+A_{I}+\frac{1}{3} A_{I}\left(A_{I}-\epsilon\right)-\frac{1}{3} \epsilon A_{I}-\frac{1}{3} \frac{1}{H} \dot{\delta}_{I}^{H}
\end{aligned}
$$

where

$$
\begin{aligned}
& A_{1}=\eta_{11}+\frac{\sin \theta}{\cos \theta} \eta_{12}, \quad A_{2}=\eta_{22}+\frac{\cos \theta}{\sin \theta} \eta_{12} \\
& A_{1}+A_{2}=\eta_{11}+\frac{\eta_{12}}{\sin \theta \cos \theta}+\eta_{22}=2 \eta_{\sigma \sigma}+\eta_{\sigma s} \frac{\cos ^{2} \theta-\sin ^{2} \theta}{\sin \theta \cos \theta}
\end{aligned}
$$

\section{APPENDIX B: DERIVATIVES}

Note that all slow-roll parameters can only be taken to be constant at the order at which they are defined, i.e., the time derivative of a first-order slow roll parameter is second order.

$$
\begin{aligned}
& \frac{1}{H} \dot{\epsilon} \cong 2 \epsilon\left(2 \epsilon-\eta_{\sigma \sigma}\right), \\
& \frac{1}{H} \dot{\eta}_{\sigma \sigma} \cong 2 \epsilon \eta_{\sigma \sigma}-2 \eta_{\sigma s}^{2}-\xi_{\sigma \sigma \sigma}^{2}, \\
& \frac{1}{H} \dot{\eta}_{\sigma s} \cong 2 \epsilon \eta_{\sigma s}+\eta_{\sigma s}\left(\eta_{\sigma \sigma}-\eta_{s s}\right)-\xi_{\sigma \sigma s}^{2}, \\
& \frac{1}{H} \dot{\eta}_{s s} \cong 2 \epsilon \eta_{s s}+2 \eta_{\sigma s}^{2}-\xi_{\sigma s s}^{2}, \\
& \frac{1}{H} \dot{\delta}_{\sigma}^{H} \cong-4 \epsilon^{2}-2 \eta_{\sigma s}^{2}+4 \epsilon \eta_{\sigma \sigma}-\xi_{\sigma \sigma \sigma}^{2} .
\end{aligned}
$$


All of the relations above will also be true to the same order if $\frac{1}{H} \frac{d}{d t}$ is replaced by $\frac{d}{d \ln k}$ at $k=a H$.

The derivative of $\epsilon^{H}$ is also required to third order for calculating the spectral tilt of $\mathcal{P}_{*}^{(0)}(40)$ to second order in slow roll, and for calculating $\beta_{*}(52)$ to second order in slow roll,

$$
\frac{d \ln \epsilon^{H}}{d \ln k} \cong 2\left(2 \epsilon-\eta_{\sigma \sigma}+\frac{7}{3} \epsilon \eta_{\sigma \sigma}-2 \epsilon^{2}-\frac{1}{3} \eta_{\sigma \sigma}^{2}-\frac{1}{3} \eta_{\sigma s}^{2}-\frac{1}{3} \xi_{\sigma \sigma \sigma}^{2}\right)
$$

\section{APPENDIX C: CALCULATING THE TILT}

In order to calculate the spectral indices of the power spectra $(58,59,60)$ we need to calculate the scale dependence of $\mathcal{P}_{*}^{(0)}$, the transfer functions and $a_{1}, a_{2}, a_{3},(41)$, all up to second order in slow roll.

We define

$$
n_{(0)} \equiv \frac{d \log \mathcal{P}_{*}^{(0)}}{d \log k}
$$

where $\mathcal{P}_{*}^{(0)}$ is defined by (40) and split the result into first and second order parts

$$
n_{(0)}^{(1)}=-6 \epsilon+2 \eta_{\sigma \sigma}, \quad n_{(0)}^{(2)}=\frac{14}{3} \epsilon^{2}+\frac{2}{3} \eta_{\sigma \sigma}^{2}+\frac{2}{3} \eta_{\sigma s}^{2}-6 \epsilon \eta_{\sigma \sigma}+\frac{2}{3} \xi_{\sigma \sigma \sigma}^{2} .
$$

The scale dependence of the transfer functions (45), where the functions $\alpha_{*}$ and $\beta_{*}$ are given by $(48,52)$ respectively and using $d \log k \simeq H\left(1-\epsilon^{H}\right) d t_{*}$ are

$$
\begin{aligned}
{\frac{\partial \log T_{\mathcal{S S}}}{d \log k}}^{(1)}= & 2 \epsilon-\eta_{\sigma \sigma}+\eta_{s s}, \\
\frac{\partial \log T_{\mathcal{S S}}{ }^{(2)}}{\partial \log k}= & -2 \epsilon^{2}-\frac{1}{3} \eta_{\sigma \sigma}^{2}+\frac{1}{3} \eta_{s s}^{2}+\frac{7}{3} \epsilon \eta_{\sigma \sigma}+\frac{1}{3} \epsilon \eta_{s s}-\frac{1}{3} \xi_{\sigma \sigma \sigma}^{2}+\frac{1}{3} \xi_{\sigma \sigma s}^{2} \\
{\frac{\partial \log T_{\mathcal{R S}}}{\partial \log k}}^{(1)}= & 2 \epsilon-\eta_{\sigma \sigma}+2 \tan \Delta \eta_{\sigma s}+\eta_{s s} \\
{\frac{\partial \log T_{\mathcal{R S}}}{\partial \log k}}^{(2)}= & -2 \epsilon^{2}-\frac{1}{3} \eta_{\sigma \sigma}^{2}-4 C \tan ^{2} \Delta \eta_{\sigma s}^{2}+\frac{1}{3} \eta_{s s}^{2}+\frac{7}{3} \epsilon \eta_{\sigma \sigma}-\tan \Delta\left(\frac{2}{3}+4 C\right) \epsilon \eta_{\sigma s}+\frac{1}{3} \epsilon \eta_{s s} \\
& +\tan \Delta\left(\frac{2}{3}+2 C\right) \eta_{\sigma \sigma} \eta_{\sigma s}+\tan \Delta\left(\frac{2}{3}-2 C\right) \eta_{\sigma s} \eta_{s s}-\frac{1}{3} \xi_{\sigma \sigma \sigma}^{2}+\frac{2}{3} \tan \Delta \xi_{\sigma \sigma s}^{2}+\frac{1}{3} \xi_{\sigma s s}^{2} .
\end{aligned}
$$

Note that the $a_{I},(41)$ are first order in slow roll, so their derivatives are second order in slow roll and can be easily calculated from (B1,B2,B3,B4).

In order to write the spectral indices in terms of observables we need to be able to relate the transfer functions and the correlation angle $(35)$. From $(58,59,60)$ it follows that at zeroth order

$$
\cos \Delta^{(0)}=\frac{T_{\mathcal{R S}}}{\sqrt{1+T_{\mathcal{R S}}^{2}}}, \quad \sin \Delta^{(0)}=\frac{1}{\sqrt{1+T_{\mathcal{R S}}^{2}}}, \quad \tan \Delta^{(0)}=\frac{1}{T}_{\mathcal{R S}}
$$

and at first order

$$
\begin{aligned}
& \cos \Delta^{(1)}=\cos \Delta^{(0)}\left[-\frac{1}{2} a_{1} \sin ^{2} \Delta+a_{2} \frac{\sin ^{3} \Delta}{\cos \Delta}+\frac{1}{2} a_{3} \sin ^{2} \Delta\right], \\
& \sin \Delta^{(1)} \equiv \sin \Delta^{(0)}\left[\frac{1}{2} a_{1} \cos ^{2} \Delta-a_{2} \sin \Delta \cos \Delta-\frac{1}{2} a_{3} \cos ^{2} \Delta\right] .
\end{aligned}
$$

Another useful equation for $T_{\mathcal{R S}}$ is

$$
\frac{1}{T_{\mathcal{R S}}}=\tan \Delta^{(0)} \simeq \tan \Delta\left(1+C\left(-2 \epsilon+\eta_{\sigma \sigma}-2 \eta_{\sigma s} \tan \Delta-\eta_{s s}\right)\right) .
$$


To find the spectral tilts we take the log derivatives of $(58,59,60)$ and substitute away the dependence on the transfer functions by replacing them with the correlation angle $(\mathrm{C} 7, \mathrm{C} 8, \mathrm{C} 9, \mathrm{C} 10)$ to find

$$
\begin{aligned}
n_{\mathcal{R}}^{(1)}= & n_{(0)}^{(1)}+2 \sin \Delta \cos \Delta \frac{d T_{\mathcal{R S}}^{(1)}}{d \log k}, \\
n_{\mathcal{R}}^{(2)}= & n_{(0)}^{(2)}+2 \sin \Delta \cos \Delta \frac{d T_{\mathcal{R S}}^{(2)}}{d \log k}-2\left(\cos \Delta \sin \Delta^{(1)}+\sin \Delta \cos { }^{(1)}\right) \frac{d T_{\mathcal{R} \mathcal{S}}^{(1)}}{d \log k} \\
& -2 a_{1} \sin ^{3} \Delta \cos \Delta \frac{d T_{\mathcal{R S}}^{(1)}}{d \log k}+2 a_{2} \sin ^{2} \Delta\left(\sin ^{2} \Delta-\cos ^{2} \Delta\right) \frac{d T_{\mathcal{R} \mathcal{S}}^{(1)}}{d \log k} \\
& +2 a_{3} \sin ^{3} \Delta \cos \Delta \frac{d T_{\mathcal{R S}}^{(1)}}{d \log k}+\sin ^{2} \Delta \frac{d a_{1}}{d \log k}+2 \sin \Delta \cos \Delta \frac{d a_{2}}{d \log k}+\cos ^{2} \Delta \frac{d a_{3}}{d \log k}, \\
n_{C}^{(1)}= & n_{(0)}^{(1)}+\frac{d \log T_{\mathcal{S S}}^{(1)}}{d \log k}+\frac{d \log T_{\mathcal{R S}}^{(1)}}{d \log k}, \\
n_{\mathcal{C}}^{(2)}= & n_{(0)}^{(2)}+\frac{d \log T_{\mathcal{S} \mathcal{S}}^{(2)}}{d \log k}+\frac{d \log T_{\mathcal{R S}}^{(2)}}{d \log k}+\tan \Delta \frac{d a_{2}}{d \log k}-a_{2} \tan \Delta \frac{d \log T_{\mathcal{R S}}^{(1)}}{d \log k}+\frac{d a_{3}}{d \log k}, \\
n_{\mathcal{S}}^{(1)}= & n_{(0)}^{(1)}+2 \frac{d \log T_{\mathcal{S} \mathcal{S}}^{(1)}}{d \log k}, \\
n_{\mathcal{S}}^{(2)}= & n_{(0)}^{(2)}+2 \frac{d \log T_{\mathcal{S} \mathcal{S}}^{(2)}}{d \log k}+\frac{d a_{3}}{d \log k} .
\end{aligned}
$$

So substituting $(\mathrm{C} 2, \mathrm{C} 3, \mathrm{C} 4, \mathrm{C} 5, \mathrm{C} 6)$ in the above six equations we find the tilts as displayed in $(63,64,65)$.

[1] D. Langlois, Phys. Rev. D 59, 123512 (1999) [arXiv:astro-ph/9906080].

[2] L. Amendola, C. Gordon, D. Wands and M. Sasaki, Phys. Rev. Lett. 88, 211302 (2002) [arXiv:astro-ph/0107089].

[3] D. Wands, N. Bartolo, S. Matarrese and A. Riotto, Phys. Rev. D 66, 043520 (2002) [arXiv:astro-ph/0205253].

[4] B. van Tent, Class. Quant. Grav. 21, 349 (2004) [arXiv:astro-ph/0307048].

[5] E. D. Stewart and D. H. Lyth, Phys. Lett. B 302, 171 (1993) [arXiv:gr-qc/9302019].

[6] M. Sasaki and E. D. Stewart, Prog. Theor. Phys. 95, 71 (1996) [arXiv:astro-ph/9507001].

[7] J. O. Gong and E. D. Stewart, Phys. Lett. B 538, 213 (2002) [arXiv:astro-ph/0202098].

[8] H. C. Lee, M. Sasaki, E. D. Stewart, T. Tanaka and S. Yokoyama, JCAP 0510, 004 (2005) [arXiv:astro-ph/0506262].

[9] C. Gordon, D. Wands, B. A. Bassett and R. Maartens, Phys. Rev. D 63, 023506 (2001) [arXiv:astro-ph/0009131].

[10] S. Groot Nibbelink and B. J. W. van Tent, arXiv:hep-ph/0011325; S. Groot Nibbelink and B. J. W. van Tent, Class. Quant. Grav. 19,613 (2002) [arXiv:hep-ph/0107272].

[11] C. T. Byrnes and D. Wands, Phys. Rev. D 73, 063509 (2006) [arXiv:astro-ph/0512195].

[12] J. E. Lidsey, A. R. Liddle, E. W. Kolb, E. J. Copeland, T. Barreiro and M. Abney, Rev. Mod. Phys. 69, 373 (1997) [arXiv:astro-ph/9508078].

[13] A. R. Liddle, A. Mazumdar and F. E. Schunck, Phys. Rev. D 58, 061301 (1998) [arXiv:astro-ph/9804177].

[14] K. A. Malik and D. Wands, Phys. Rev. D 59, 123501 (1999) [arXiv:astro-ph/9812204].

[15] E. J. Copeland, A. Mazumdar and N. J. Nunes, Phys. Rev. D 60, 083506 (1999) [arXiv:astro-ph/9904309].

[16] K. Enqvist and M. S. Sloth, Nucl. Phys. B 626, 395 (2002) [arXiv:hep-ph/0109214]; D. H. Lyth and D. Wands, Phys. Lett. B 524, 5 (2002) [arXiv:hep-ph/0110002]; T. Moroi and T. Takahashi, Phys. Lett. B 522, 215 (2001) [Erratum-ibid. B 539, 303 (2002)] [arXiv:hep-ph/0110096].

[17] A. Taruya and Y. Nambu, Phys. Lett. B 428, 37 (1998) [arXiv:gr-qc/9709035].

[18] N. Bartolo, S. Matarrese and A. Riotto, Phys. Rev. D 64, 083514 (2001) [arXiv:astro-ph/0106022].

[19] B. A. Bassett, S. Tsujikawa and D. Wands, arXiv:astro-ph/0507632.

[20] J. Valiviita and V. Muhonen, Phys. Rev. Lett. 91, 131302 (2003) [arXiv:astro-ph/0304175]; H. Kurki-Suonio, V. Muhonen and J. Valiviita, Phys. Rev. D 71, 063005 (2005) [arXiv:astro-ph/0412439].

[21] H. V. Peiris et al., Astrophys. J. Suppl. 148, 213 (2003) [arXiv:astro-ph/0302225].

[22] M. Cortes and A. R. Liddle, [arXiv:astro-ph/0603016].

[23] A. Kosowsky and M. S. Turner, Phys. Rev. D 52, 1739 (1995) [arXiv:astro-ph/9504071].

[24] D. H. Lyth, C. Ungarelli and D. Wands, Phys. Rev. D 67, 023503 (2003) [arXiv:astro-ph/0208055].

[25] E. W. Kolb, A. Riotto and A. Vallinotto, Phys. Rev. D 71, 043513 (2005) [arXiv:astro-ph/0410546].

[26] G. N. Felder, L. Kofman and A. D. Linde, Phys. Rev. D 59, 123523 (1999) [arXiv:hep-ph/9812289].

[27] N. Bartolo, S. Matarrese and A. Riotto, Phys. Rev. D 64, 123504 (2001) [arXiv:astro-ph/0107502]. 